\title{
DESVELANDO A IMPLEMENTAÇÃO DA SISTEMATIZAÇÃO DA ASSISTÊNCIA DE ENFERMAGEM*
}

\section{Resumo}

Trata-se de um estudo da revisão de literatura nacional acerca da sistematização da assistência de enfermagem - SAE, cujos objetivos foram identificar as dificuldades encontradas na implementação dessa prática e os fatores que interferem prejudicando sua implementação. Foram acionadas as bases de dados MEDLINE, LILACS e BDENF e levantados seis trabalhos publicados em periódicos de enfermagem, realizados nos últimos cinco anos. Os resultados apontam diversas dificuldades na implementação da SAE e fatores de ordens distintas interferindo negativamente na sua operacionalização. Considerando a relevância dessa metodologia assistencial para a valorização da enfermagem profissional, é preciso refletir/discutir sobre suas dificuldades práticas, para que se possa superá-las, tornando-a uma atividade prazerosa, capaz de conferir autonomia para o enfermeiro e qualidade na assistência ao paciente.

Descritores: enfermagem; processo de enfermagem; ensino superior

\begin{abstract}
This study has reviewed the national literature regarding nursing assistance systematization (NAS), with the aim of identifying the difficulties implementing this practice and the factors that interfere with and harm its implementation. The MEDLINE, LILACS, and BDENF databases have been utilized and six studies published in nursing periodicals in the last five years have been surveyed. The results indicate several difficulties implementing the NAS and several factors that interfere negatively with its implementation. Considering the importance of this assistance methodology for valuing professional nursing, it is necessary to reflect on/discuss its practical difficulties so that we can overcome them, making it a pleasurable activity capable of providing nurses with autonomy and providing patients with quality assistance.

Descriptors: nursing; nursing process; higher education

Title: Clarifying the Implementation of the Nursing assistance systemization
\end{abstract}

\section{Resumen}

Se trata de un estudio de la revisión de la literatura nacional al respecto de la sistematización de la asistencia de enfermería - SAE, cuyos objetivos fueron identificar las dificultades encontradas en la implementación de esa práctica y los factores que contribuyen para dificultar su implementación. Se consultaron las bases de datos MEDLINE, LILACS y BDENF, y levantados sus trabajos publicados en periódicos de enfermería realizados en los últimos cinco años. Los resultados indican diversas dificultades en la implementación de la SAE y factores de órdenes distintas que interfieren negativamente en su operacionalización. Considerando la relevancia de esta metodología asistencial para la valorización de la enfermería profesional, es necesario reflexionar/discutir sobre sus dificultades prácticas, para que pueda ser posible superarlas, tornándola una actividad satisfactoria, capaz de proporcionar autonomía para el enfermero y la cualidad en la asistencia al paciente.

Descriptores: enfermería; proceso de enfermería; enseñanza superior

Título: Desvelando la implementación de la sistematización de la asistencia de Enfermería

\section{Introdução}

A evolução da enfermagem e sua consolidação enquanto ciência é caracterizada pela construção de um corpo de conhecimento próprio no decorrer de sua história, mas especificamente a partir da década de 50 . Nos anos 70 houve uma preocupação das enfermeiras com o desenvolvimento de teorias de enfermagem, como um meio de estabelecer a enfermagem como profissão(1).

Dentro desse corpo de conhecimentos, baseado em teorias de enfermagem, surge o processo de enfermagem (PE), sendo uma representação do mecanismo por meio do qual esses conhecimentos são aplicados na prática profissional(2)

O PE já vinha sendo aplicado nos Estados Unidos e Reino Unido, quando na década de 70, chegou ao Brasil invadindo as escolas de enfermagem e contribuindo para a teoria de enfermagem de Wanda de Aguiar Horta(2).

O PE recebe várias definições de acordo com muitos autores e teorias, sendo que, em cada uma delas segue um modelo e é fundamentado de acordo com os conceitos, pressupostos e proposições próprias dessas teorias. $\mathrm{Na}$ verdade, o PE pode ser entendido como a aplicação prática de uma teoria de enfermagem no cotidiano da assistência de enfermagem aos pacientes.

O PE trata-se de uma forma organizada de cuidar do paciente, seguindo alguns passos previamente estabelecidos (coleta de dados, diagnóstico, planejamento, implementação e avaliação dos resultados) ${ }^{(3)}$. É um método a ser seguido a fim de alcançar os objetivos desejados em relação à assistência de enfermagem, o qual baseia-se num modelo assistencial e deve levar em conta a especialidade à que está sendo dirigido (cardiologia, pediatria, etc).

Para uma melhor compreensão desse conceito, cabe aqui uma breve explicação sobre o que é um método. De maneira geral, é entendido como uma forma sistemática e ordenada de delimitar problemas, fazer e executar planos para resolvê-los, mesmo que seja necessário delegar a outros a execução desses planos, e exige como última etapa a avaliação da eficácia na resolução dos problemas ${ }^{(4)}$.

Diante dessas considerações, a enfermagem deve privilegiar suas ações específicas/próprias junto ao cliente e atuar como parceira dos demais profissionais, não apenas como suporte de ações médicas e administrativo-burocráticas, mas compartilhando seus saberes no atendimento às necessidades do paciente ${ }^{(5,6)}$.

Há uma interdependência entre o trabalho médico e de enfermagem, porém, cada disciplina exerce funções específicas e tem uma visão diferente acerca do ser humano, enquanto objeto de estudo/trabalho que requer atenção de ambos.

Dentre as vantagens de utilizar-se um método como uma estratégia para a assistência de enfermagem estão a sustentação de um padrão mínimo de qualidade da assistência e, um melhor sistema de registro de informações sobre o paciente(5).

A sistematização da assistência de enfermagem - SAE, através do processo de enfermagem, tem sido alvo de trabalhos

\footnotetext{
* Trabalho apresentado à disciplina Processo de cuidar em enfermagem da Pós-Graduação em Enfermagem da FCM - UNICAMP. **Enfermeira. Especialista em Formação Profissional na Àrea da Saúde - Enfermagem, Mestranda em Enfermagem na Pós-Graduação em Enfermagem da FCM -UNICAMP.

E-mail do autor: patymadale@yahoo.com.br
} 
acadêmicos e de experiências práticas em algumas instituições. No Brasil a sistematização "tem sido discutida, desde o início da década de 70 , visando à eficácia da assistência, ampliação e definição do espaço da enfermagem na equipe de saúde"(3:5).

Após a iniciativa e investimento do COREN-SP, em 1999, de implantar o PE de forma definitiva nas instituições de saúde públicas e privadas de todo o Estado, a Resolução COFEN $n^{\circ}$ 272/2002, surgiu como apoio legal para a implementação dessa prática em âmbito nacional, dispondo sobre a SAE nas instituições de saúde brasileiras ${ }^{(7)}$. Ela trata no seu Art. $1^{\circ}$ sobre a função privativa do enfermeiro de implantar, planejar, organizar, executar e avaliar o PE, o qual compreende: histórico, exame físico, diagnóstico, prescrição e evolução de enfermagem.

As dificuldades encontradas na implementação do PE são apontadas por acadêmicos de enfermagem, enfermeiros e docentes em diversos trabalhos, o que justifica a relevância deste estudo para que se possa pensar em superá-las no cotidiano da prática da enfermagem assistencial.

Portanto, este estudo tem como objetivo identificar as dificuldades encontradas na implementação da SAE e os fatores que interferem prejudicando sua implementação, nos trabalhos nacionais publicados nos últimos cinco anos.

\section{Metodologia}

Para realizar este estudo de revisão de literatura, primeiramente foi feito um levantamento bibliográfico acerca da temática da SAE, utilizando as bases de dados do Lilacs, Medline e Bdenf, no Sistema Bireme.

Para proceder a busca das referências bibliográficas nestas bases de dados, foram utilizados como palavras-chave: sistematização, assistência, enfermagem e processo de enfermagem. A partir dessa busca realizou-se a leitura de todos os títulos e resumos das referências bibliográficas identificadas nas bases de dados.

$\mathrm{Na}$ etapa subsequente foram selecionadas as referências bibliográficas de interesse para este estudo, considerando como critérios: ser um trabalho desenvolvido no âmbito nacional; ter sido publicado nos últimos cinco anos; abordar no resumo do trabalho as dificuldades na implementação da SAE/processo de enfermagem e/ou os fatores que interferem prejudicando a sua operacionalização.

As referências bibliográficas selecionadas são de trabalhos publicados em periódicos de enfermagem nacional (Revista Gaúcha de Enfermagem, Acta Paulista de Enfermagem, Latino-Americana de Enfermagem, Baiana de Enfermagem e Nursing), caracterizados como trabalhos de pesquisa.

Os estudos foram categorizados em Trabalhos A, B, C, $D, E$ e $F$ para facilitar a apresentação dos dados. Cada categoria representa um estudo publicado num periódico e pode ser entendido como:

Trabalho A, pesquisa realizada com enfermeiras chefes em 18 hospitais de São Paulo(8).

Trabalho $B$, pesquisa realizada com enfermeiros que trabalham nas unidades de internação de clínicas médica e cirúrgica, num hospital do interior de São Paulo ${ }^{(9)}$.

Trabalho C, uma pesquisa realizada com acadêmicos e enfermeiros de um hospital e Universidade do Rio Grande do Sul(10).

Trabalho $D$, pesquisa realizada com enfermeiros da unidade de queimados de um hospital do interior de São Paulo(11).

Trabalho E, pesquisa desenvolvida com enfermeiros que trabalham em uma UTI de um hospital geral de grande porte da região sul do Rio Grande do Sul(12).

Trabalho $F$, pesquisa realizada com enfermeiros que trabalham em UTIs gerais de dois hospitais, um hospital universitário e o outro um hospital-escola privado, situados em Salvador/Bahia(13).
Para este estudo foi considerado que a dificuldade na implementação da SAE pode ser entendida como aquilo que é difícil de colocar em prática, por exemplo, pode ser difícil realizar a ausculta pulmonar no exame físico ou pode ser um obstáculo fazer o diagnóstico de enfermagem e prescrever cuidados. Por outro lado, os fatores que interferem prejudicando a implementação da SAE é aquilo que favorece uma dificuldade, exemplificando, a falta de tempo ou o preparo inadequado na graduação.

\section{Resultados e Discussões}

A apresentação dos dados levantados nas pesquisas analisadas e seus respectivos resultados e discussões serão a seguir comentados.

\subsection{Dificuldades encontradas na implementação da SAE}

É importante esclarecer que, em todos os trabalhos ficou muitas vezes difícil distinguir as dificuldades apresentadas na implementação da SAE e os fatores que interferem prejudicando sua implementação. Portanto, os dados foram agrupados nos quadros, segundo o entendimento da autora acerca dessa distinção de termos.

O Trabalho $\mathrm{B}$ apontou o maior número de dificuldades na operacionalização da SAE, diferentemente do Trabalho A, cujos dados foram interpretados como fatores que estavam prejudicando a implementação da metodologia.

A operacionalização da proposta da SAE por meio do processo de enfermagem tem esbarrado em muitas dificuldades, prevalecendo na prática clínica a organização do cuidado centrado em tarefas, ou seja, o que importa é a realização da ação(2), o que foi identificado nos trabalhos $D$ e E.

Publicações nacionais menos recentes, também destacam várias dificuldades na operacionalização da SAE, algumas já citadas pelos trabalhos desta revisão(14,15).

Há estudos que vão além e retratam que existem dificuldades e incompatibilidades dentro do próprio ensino do P.E. nas escolas de graduação em enfermagem ${ }^{(16-18)}$. Isso pode de certa forma refletir e justificar algumas dificuldades apresentadas posteriormente na atividade do profissional e várias delas podem ser observadas no quadro 1 , exemplificando, a dificuldade em realizar o exame físico.

\subsection{Fatores que interferem prejudicando a implementação da SAE}

Para alguns autores existem ordens diferentes de fatores que interferem na aplicação do PE e esses se inter-relacionam. Alguns desses fatores estão no âmbito da organização (políticas, normas, objetivos dos serviços, muitas vezes estabelecidos por médicos e administradores sem a participação dos enfermeiros), outros fatores fazem parte do próprio quotidiano desses profissionais (atitudes, crenças, valores, habilidades técnicas e intelectuais), para os quais muitas vezes se busca explicação nas deficiências do ensino formal e na sua relação com a prática. Um outro fator é a maneira como o processo tem sido operacionalizado nos serviços - uma imposição da chefia de enfermagem que valoriza mais a documentação à implementação dessa metodologia de forma efetiva na prática ${ }^{(2)}$.

Os fatores identificados nos trabalhos foram classificados neste estudo como sendo de ordem: Pessol/Profissional (quadro 2), Organizacional (quadro 3), relacionados ao Modelo Teórico (quadro 4) e outros (quadro 5).

É possível identificar que são muitos os fatores de ordem Pessoal/Profissional que prejudicam a implementação da SAE, com destaque, o preparo inadequado na graduação/despreparo do pessoal, presentes em quatro dos trabalhos pesquisados.

Alguns autores, observando a formação do enfermeiro, confirmam que o aluno tem saído da graduação sem o amplo conhecimento necessário para colocar em prática o método específico de sua especialidade, que qualifica sua atividade junto aos pacientes, transmitindo-Ihes confiança e segurança ${ }^{(9)}$. 


\begin{tabular}{|c|c|c|c|c|c|c|}
\hline \multirow{2}{*}{ DIFICULDADES } & \multicolumn{6}{|c|}{ Trabalhos } \\
\hline & $A$ & $B$ & $\mathrm{C}$ & $\mathrm{D}$ & $E$ & $\mathrm{~F}$ \\
\hline Realizar o exame físico & & * & & & & \\
\hline Adequar o exame físico a doença apresentada pelo paciente & & * & & & & \\
\hline Realizar ausculta pulmonar e cardíaca & & * & & & & \\
\hline Necessidade de aprofundamento teórico e falta de prática & & * & & & & \\
\hline Cumprimento da prescrição de enf. pela própria equipe de enf. & & * & & & & \\
\hline Descontinuidade da implementação da assistência entre turnos & & * & & & & \\
\hline Alguns profissionais não lêem o que está prescrito & & * & & & & \\
\hline Falta de conhecimento mesmo procurando por ele & & * & & & & \\
\hline Insegurança & & & * & & & \\
\hline Prescrição de enfermagem sem avaliação prévia do paciente & & & & * & & \\
\hline Cuidados prescritos ou não, são realizados rotineiramente & & & & * & & \\
\hline Não saber realizar o processo de enfermagem & & & & & * & \\
\hline Dificuldade de elaborar a prescrição de enfermagem & & & & & & * \\
\hline Falta de objetividade na elaboração da prescrição & & & & & & * \\
\hline Não checagem dos cuidados na prescrição & & & & & & * \\
\hline Não há evolução de enfermagem mas descrição do estado do paciente e dos cuidados executados & & & & & & * \\
\hline Falta de objetividade no levantamento de problemas & & & & & & * \\
\hline
\end{tabular}

Quadro 1 - Distribuição das dificuldades na implementação da SAE, em seis estudos publicados em periódicos nacionais, nos últimos cinco anos.

\begin{tabular}{|c|c|c|c|c|c|c|}
\hline \multirow{2}{*}{ Fatores Pessoais/Profissionais que interferem prejudicando a implementação da SAE } & \multicolumn{6}{|c|}{ Trabalhos } \\
\hline & A & $\mathrm{B}$ & $\mathrm{C}$ & $\mathrm{D}$ & $\mathrm{E}$ & $\mathrm{F}$ \\
\hline Preparo inadequado na graduação/despreparo do pessoal & * & * & * & & & * \\
\hline Falta de comprometimento, envolvimento e responsabilidade de enfermeiros/desinteresse e desmotivação & * & & & & & * \\
\hline Perfil e postura inadequados de algumas profissionais & * & & & & & \\
\hline Falta de liderança e de organização de enfermeiras & * & & & & & \\
\hline Pouca disponibilidade de enfermeiras e excesso de trabalho & * & & & & * & \\
\hline Falhas no desempenho e falta de confiança de enfermeiras em seu trabalho & * & & & & & \\
\hline Desvalorização do trabalho e desconsideração sobre a importância da SAE por parte das enfermeiras & * & & & & & \\
\hline Problemas de relacionamento interpessoal & * & & & & & \\
\hline O enfermeiro não gosta de receber ordem de outro enfermeiro & & * & & & & \\
\hline Ausência de definição de papéis do quadro de enfermagem & & * & & & & \\
\hline Falta de leitura da prescrição de enfermagem pela equipe & & * & & & & \\
\hline Falta de conscientização da equipe de enfermagem & & * & & & & \\
\hline Falta de treinamento da equipe de enfermagem & & * & & & & \\
\hline Falta de trabalho em equipe para padronizar a assistência & & & * & & & \\
\hline Falta de integração e resistência de enfermeiras em envolver-se com propostas de melhoria da assistência & * & & & & & \\
\hline Estresse & & & & & & * \\
\hline Questões salariais & & & & & & * \\
\hline
\end{tabular}

Quadro 2 - Distribuição dos fatores Pessoais/Profissionais que interferem prejudicando a implementação da SAE, em seis estudos publicados em periódicos nacionais, nos últimos cinco anos.

\begin{tabular}{|c|c|c|c|c|c|c|}
\hline \multirow{2}{*}{ Fatores Organizacionais que interferem prejudicando a implementação da SAE } & \multicolumn{6}{|c|}{ Trabalhos } \\
\hline & A & B & C & $\mathrm{D}$ & $\mathrm{E}$ & $\mathrm{F}$ \\
\hline Carência de pessoal de enfermagem/enfermeiros & * & * & * & & & * \\
\hline Responsabilidade por duas enfermarias & & * & & & & \\
\hline Atividades administrativas concomitantes com as assistenciais & & & * & & & \\
\hline Aplicação do processo como mais uma tarefa & & & & * & & \\
\hline $\begin{array}{l}\text { A instituição como organização burocrática não espera que seja realizado outro } \\
\text { cuidado, além do estabelecido pelo médico }\end{array}$ & & & & * & & \\
\hline Falta de vontade das chefias e da instituição & & & & & & * \\
\hline $\begin{array}{l}\text { Falta de material e/ou equipamento como sintoma da "falta de vontade das } \\
\text { chefias e instituições" }\end{array}$ & & & & & & * \\
\hline Inadequação da estrutura física da Unidade & & & & & & * \\
\hline Falhas na estrutura administrativa da instituição & * & & & & & \\
\hline "Ser instituição pública" & & & & & & * \\
\hline Baixa eficiência de serviços de apoio (laboratório, etc) & & & & & & * \\
\hline
\end{tabular}

Quadro 3 - Distribuição dos fatores Organizacionais que interferem prejudicando a implementação da SAE, em seis estudos publicados em periódicos nacionais, nos últimos cinco anos. 


\begin{tabular}{|l|c|c|c|c|c|c|}
\hline \multirow{2}{*}{$\begin{array}{c}\text { Fatores relacionados ao Modelo Teórico } \\
\text { que interferem prejudicando a } \\
\text { implementação da SAE }\end{array}$} & \multicolumn{5}{|c|}{ Trabalhos } \\
\cline { 2 - 7 } & A & B & C & D & E & F \\
\hline $\begin{array}{l}\text { Ausência de etapas (histórico de } \\
\text { enfermagem e diagnóstico) que embasem } \\
\text { a elaboração da prescrição de enfermagem }\end{array}$ & & & & & & * \\
\hline $\begin{array}{l}\text { Lacuna entre o histórico e a prescrição de } \\
\text { enfermagem }\end{array}$ & & & & & & $*$ \\
\hline $\begin{array}{l}\text { Levantamento de problemas apenas como } \\
\text { elaboração mental }\end{array}$ & & & & & & $*$ \\
\hline $\begin{array}{l}\text { Complexidade do Processo de } \\
\text { Enfermagem }\end{array}$ & & & & & & $*$ \\
\hline
\end{tabular}

Quadro 4 - Distribuição dos fatores relacionados ao modelo teórico que interferem prejudicando a implementação da SAE, em seis estudos publicados em periódicos nacionais, nos últimos cinco anos.

\begin{tabular}{|l|c|c|c|c|c|c|}
\hline \multirow{2}{*}{$\begin{array}{c}\text { Outros fatores que interferem prejudicando } \\
\text { a implementação da SAE }\end{array}$} & \multicolumn{7}{|c|}{ Trabalhos } \\
\cline { 2 - 7 } & A & B & C & D & E & F \\
\hline $\begin{array}{l}\text { Paciente em estado de inconsciência e } \\
\text { instabilidade }\end{array}$ & & & & & $*$ & $*$ \\
\hline Falta de tempo & & $*$ & $*$ & & * & * \\
\hline Morosidade & & & & & & $*$ \\
\hline $\begin{array}{l}\text { Recursos financeiros insuficientes para o } \\
\text { aprimoramento profissional de enfermeiras }\end{array}$ & $*$ & & & & & \\
\hline
\end{tabular}

Quadro 5 - Distribuição de outros fatores que interferem prejudicando a implementação da SAE, em seis estudos publicados em periódicos nacionais, nos últimos cinco anos.

Em 1989 já se apresentava dentre os fatores que mais freqüentemente dificultam a utilização do $\mathrm{PE}$, a falta de preparo dos enfermeiros sobre o método ${ }^{(14)}$. Talvez seja pertinente questionar até que ponto as instituições de ensino de graduação têm discutido e implantado medidas para resolver esse problema.

Outra autora também afirma que um dos problemas para a implementação da SAE é que a maioria dos profissionais de enfermagem desconhece o assunto, por deficiência do processo de formação(3).

Um estudo realizado em 2001 contribui apontando que a efetiva implantação do PE e sua continuidade com sucesso, exige que o enfermeiro esteja preparado com conhecimentos científicos e constante atualização(12).

Publicações menos recentes também citam alguns fatores que têm dificultado a implementação dessa metodologia, alguns inclusive presentes nos trabalhos analisados neste estudo(14-18).

Observa-se que muitos dos fatores que prejudicam a implementação da SAE apontados nos estudos desta revisão se apresentam, no âmbito da organização, destacando-se a carência de pessoal de enfermagem/enfermeiros, identificada em quatro dos trabalhos em estudo.

Assim, é preciso salientar a responsabilidade das instituições propiciarem todas as condições necessárias ao enfermeiro para executar de forma efetiva o planejamento da assistência, pois, não basta fazer, é preciso fazê-lo bem; não basta exigir, é preciso oferecer condições ${ }^{(19)}$.

É preciso considerar que "para a implementação do processo de enfermagem há pelo menos duas barreiras iniciais a serem transpostas: uma relacionada à escolha, interpretação e aplicação do modelo conceitual, e a outra a sua operacionalização no contexto da prática"(2:50).
O quadro 4 mostra que apenas o Trabalho $F$ apontou fatores relacionados ao modelo teórico que prejudicam a implementação da SAE, estando todos ligados apenas a etapa de aplicação do modelo conceitual. Pode-se até indagar se as demais tentativas de implementação têm procurado seguir algum modelo conceitual ou são realizadas empiricamente, sem um modelo teórico que subsidie essa prática.

A falta de tempo foi destaque entre os outros fatores que prejudicam a implementação da SAE, apontada em quatro dos trabalhos em estudo.

É possível identificar através dos quadros 2 a 5 que as dificuldades nessa implementação têm sido atribuídas a diversos fatores, apresentados pelos seis trabalhos em estudo, porém, alguns são mais representativos, encontrando-se o maior número de fatores no âmbito Pessoal/Profissional e Organizacional. Assim sendo, precisam ser considerados para que seja possível a implementação dessa prática nos locais onde foram realizadas as pesquisas.

Alguns autores fazem sugestões para se alcançar a implementação da SAE efetivamente ou para superar as dificuldades encontradas na implementação dessa proposta. Nesse sentido, escrevem que para o sucesso da SAE deve haver uma parceria com a educação continuada a fim de suprir as dificuldades reais dos profissionais, com enfermeiros responsáveis/competentes, que tenham referenciais teóricos, objetivos assistenciais e avaliem constantemente essas práxis $^{(9)}$. Além disso, apontam a necessidade de se promover uma ampla discussão sobre o assunto nas Escolas de Enfermagem, a fim de determinar o referencial teórico que embasará o PE e definir a metodologia a ser empregada no ensino do mesmo ${ }^{(10)}$. Para vários autores um dos caminhos para a implementação eficaz é a integração docenteassistencial, ou seja, a integração entre as faculdades e as instituições de saúde que servem de campo de estudo para os futuros enfermeiros ${ }^{(3,12,13)}$.

Para uma efetiva implantação do PE, existem autores que consideram necessário haver primeiro um comprometimento da chefia de enfermagem com a proposta, promovendo reuniões e elaborando um plano de ação que incluiria: a sensibilização da equipe para a importância dessa metodologia; o desenvolvimento de um estudo aprofundado do tema com o envolvimento de toda a equipe; e a construção coletiva dos meios para viabilizar a execução do processo. Esses autores esclarecem que, o plano de ação viria garantir e assegurar que os objetivos fossem direcionados para viabilizar a implementação do $\mathrm{PE}$, tendo como primeiro passo a sensibilização dos enfermeiros ${ }^{(12)}$.

\section{Considerações Finais}

A temática da sistematização da assistência de enfermagem é atual embora seja discutida no Brasil desde a década de 70, com tentativas de implementação em diversas especialidades e contextos (hospitalar, ambulatorial, creche, etc).

No entanto, percebe-se que essas tentativas nem sempre atingem o êxito desejado, pois "esbarram" em dificuldades das mais variadas origens, tornando a implementação da SAE um processo desestimulador e muitas vezes inviável na prática dos profissionais de enfermagem. Outras vezes ela persiste basicamente como uma atividade burocrática, perdendo toda a sua essência.

As facilidades e dificuldades na implementação do processo de enfermagem devem ser analisadas pela equipe de enfermagem, uma vez que cada instituição possui suas peculiaridades, a fim de que o método seja implantado com conhecimento da situação e com metas possíveis de serem alcançadas ${ }^{(14)}$

A valorização da enfermagem enquanto profissão depende também da postura do profissional frente aos problemas que emergem da sua prática. O enfermeiro precisa 
ser autêntico e conquistar o seu espaço com mérito, através do uso do seu conhecimento científico específico, que pode ter na SAE a autonomia necessária para desenvolver um trabalho consciente, eficiente e gratificante do ponto de vista de resultados positivos na assistência prestada.

Considerando a relevância da SAE para a valorização da enfermagem, embasada por corpo de conhecimento científico próprio, é necessário refletir e discutir sobre as dificuldades levantadas neste estudo, bem como os fatores que desencadeiam e sustentam tais dificuldades, para que se possa superá-las, tornando sua implementação uma atividade prazerosa e principalmente, garantindo ao paciente uma assistência com mais qualidade.

\section{Referências}

1. Ângelo $M$, Forcella HT, Fukuda IMK. Do empirismo à ciência: a evolução do conhecimento de enfermagem. Revista da Escola de Enfermagem da USP, São Paulo 1995 ago; 29(2):211-23.

2. Rossi LA, Casagrande LDR. Processo de enfermagem: a ideologia da rotina e a utopia do cuidado individualizado. In: Cianciarullo TI Gualda DMR, Melleiro MM, Anabuki MH, organizadores. Sistema de assistência de enfermagem: evolução e tendências. São Paulo: Ícone; 2001. p.41-62.

3. Conselho Regional de Enfermagem (SP). Sistematização da assistência melhora qualidade do cuidar. Publicação Oficial Bimestral do CORENSP, São Paulo 1999 mai/jun;22(5):5.

4. Leopardi MT. Teorias em enfermagem: instrumentos para a prática. Florianópolis (SC): Papa-Livros;1999.226p.

5. Leopardi MT. O método como objetificação científica na assistência da enfermagem. Texto e Contexto Enfermagem, Florianópolis (SC) 1995 jan/jun; 4(1):9-18.

6. Leopardi MT, Santos I, Sena RR. Tendências de enfermagem no Brasi - tecnologias do cuidado e valor da vida. In: CBEn. Anais do $51^{\circ}$ Congresso Brasileiro de Enfermagem: Enfermagem: situando-se no mundo e construindo o futuro; 1999 out 2-7; Florianópolis (SC), Brasil. Florianópolis (SC): ABEn; 2000. p.147-73.

7. Conselho Regional de Enfermagem (SP). COREN-SP monta estratégia para implantar processo de enfermagem. Publicação Oficial Bimestra do COREN-SP, São Paulo 1999 maio/jun;22:(5):4.

8. Monte ADAS, Adami NP, Barros ALBL. Métodos avaliativos da assistência de enfermagem em instituições hospitalares. Acta Paulista de Enfermagem, São Paulo 2001 jan/abr;14(1):89-97.
9. Thomaz VA, Guidardello EB. Sistematização da assistência de enfermagem: problemas identificados pelos enfermeiros. Nursing: Revista Técnica de Enfermagem, São Paulo 2002 nov; 54(11):28-33.

10. Thofehrn MB, Traversi MS, Muniz RM, Duarte AC, Leite MP. O processo de enfermagem no cotidiano dos acadêmicos de enfermagem e enfermeiros. Revista Gaúcha de Enfermagem, Porto Alegre (RS) 1999 jan; 20(1):69-79.

11. Rossi LA, Casagrande LDR. O processo de enfermagem em uma unidade de queimados: um estudo etnográfico. Revista Lat-Am Enfermagem, Ribeirão Preto (SP) 2001 set; 9(5):39-46.

12. Matté VM, Thofehrn MB, Muniz RM. Opinião dos enfermeiros quanto à aplicabilidade do processo de enfermagem em unidade de tratamento intensivo. Revista Gaúcha de Enfermagem, Porto Alegre (RS) 2001 jan; 22(1):101-21

13. Santos JF, Ramos TAG. Implementação da metodologia de assistência de enfermagem em UTI(s) - como está e quais os fatores intervenientes. Revista Baiana de Enfermagem, Salvador (BA) 1998 abr;11(1):47-61.

14. Souza MF. Método de assistência de enfermagem. In: Anais do Jubileu de Ouro do Curso de Graduação em Enfermagem da Escola Paulista de Medicina; 1989 jun 5-9; São Paulo, Brasil. São Paulo: Escola Paulista de Medicina, Departamento de Enfermagem;1989.p.235-40.

15. Araújo IEM, Lamas JLT, Ceolim MF, Bajay HM. Sistematização da assistência de enfermagem em uma unidade de internação: desenvolvimento e implementação de roteiro direcionador; relato de experiência. Acta Paulista de Enfermagem, São Paulo 1996 jan/abr; 9(1):18-27.

16. Barros ALBL. O trabalho docente assistencial de enfermagem no hospital São Paulo da UNIFESP/EPM [tese de Livre Docência]. São Paulo: Escola Paulista de Medicina, Universidade Federal de São Paulo;1998. $70 \mathrm{f}$.

17. Dell'acqua MCQ, Miyadahira AMK. Processo de enfermagem: fatores que dificultam e os que facilitam o ensino. Revista da Escola de Enfermagem da USP, São Paulo 2000 dez; 34(4):383-9.

18. Dell'acqua MCQ, Miyadahira AMK. Ensino do processo de enfermagem nas escolas de graduação em enfermagem do estado de São Paulo. Revista Lat-Am Enfermagem, Ribeirão Preto (SP) 2002 mar/abr; 10(2):185-91.

19. Ferreira NMLA. Sistematização da assistência de enfermagem importância para a profissão e responsabilidade no preparo do enfermeiro. Acta Paulista de Enfermagem, São Paulo 1990 set; 3(3):79-84.

Data de Recebimento: 19/02/2004

Data de Aprovação: 22/12/2004 